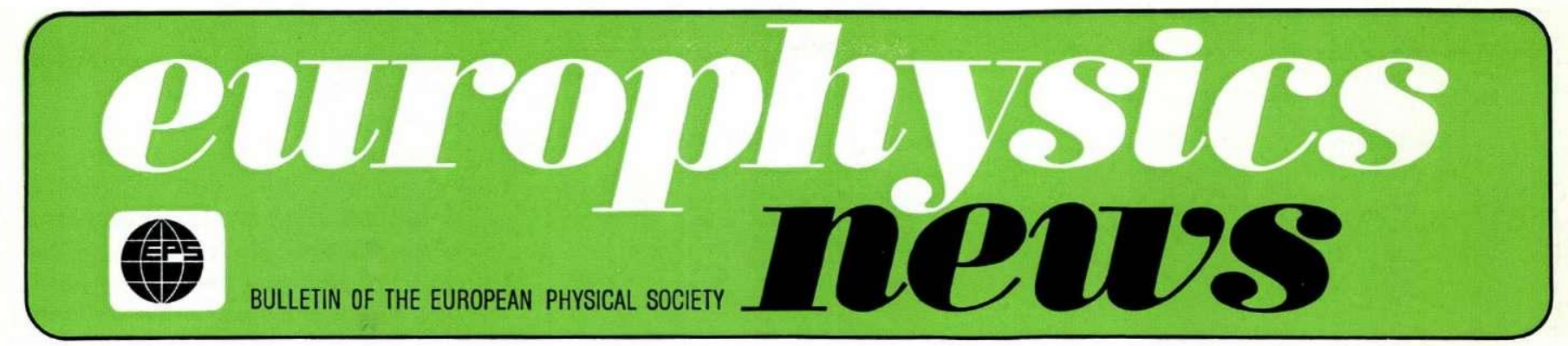

\section{8 \\ Hewlett-Packard Europhysics Prize}

The Hewlett-Packard Europhysics Prize, awarded annually for outstanding achievement in Solid State Physics leading to advances in the fields of electronic, electrical or materials engineering, has been won this year by Professor Zhores Ivanovich Alferov of the A.F. Ioffe Physico-Technical Institute in Leningrad.

The award is made for his contributions to our understanding and the practical realisation of heterojunctions - semi-conductors comprising two dissimilar compounds with capabilities that can be far greater than those obtainable with "conventional" semi-conductor materials.

Alferov and others were able to demonstrate theoretically, some $\mathbf{1 5}$ years ago, the principle that it should be possible to match crystal zones formed within mixed compounds, in order to produce very large fluxes of electrons or light energy. This opened the way to making semi-conductors that as detectors, were much more sensitive and covered a greater range than before, and when used as emitters or convertors had a very much greater power per unit volume than had previously been achieved.

The major problem was to produce an effective realisation of the system. For a number of years, it seemed that the practical application of the discovery would be severely limited as with all the known combinations of semi-conductor materials, even the most minute traces of impurities destroyed the property. This remained the position until Alferov and his group discovered an ideal partner for the semi-conductor gallium arsenide, viz. aluminium arsenide. These two substances form a solid solution together, with properties than can be readily controlled.

Alferov had already made a con-

cont. on page 12

\title{
Central Bureau for Nuclear Measurements, Geel
}

\author{
W. Bambynek, I. V. Mitchell, H. Weigmann
}

The Treaty of Rome (1957) setting up the European Atomic Energy Community (Euratom) provided for the foundation of a Joint Research Centre (JRC) which currently comprises four establishments of which the Central Bureau for Nuclear Measurements (CBNM) is one. It is located at Geel in Belgium $(5 \mathrm{~km}$ from the Belgian Nuclear Studies Centre, SCK/CEN at Mol) where site work began in 1960 . The other establishments of the JRC are at Karlsruhe (German Federal Republic), Petten (The Netherlands) and Ispra (Italy).

In the initial Euratom research programme, the CBNM's task was defined as that of a bureau of standards specializing in nuclear measurements. For this the preparation and definition of the basic necessary standards are required and they in turn, demand continuous efforts to improve measuring instruments and methods. Additional fields of activity pertain to radioactivity, isotopic composition and the preparation of well-defined samples. Specialized laboratories for chemistry, classical metrology and electronics support the nuclear activities.

To date, the main objective has been to measure nuclear properties for the development, running and safeguarding of nuclear energy installations and to advance the knowledge of the underlying physics. The fabrication and characterization of samples and targets for use in nuclear measurements and the preparation of nuclear reference material is a further task of the CBNM.

At the present time, the CBNM has a total of 200 staff members, $22 \%$ of whom are university trained graduates; $45 \%$ are scientific-technical staff and the others belong to the workshop, administration and infrastructure. The personnel are drawn from all nine member states of the European Communities to form a truly multinational scientific community.

The technical proposals and the execution of the scientific programme are examined regularly by international advisory committees whose members are made up of specialists provided by the national governments of the Communities.

\section{Neutron Data Measurements}

The programme of the Neutron Data Group of CBNM is mainly concerned with the measurement of differential neutron data for applied purposes. Neutron data are needed for reactor design and reactor safety calculations for waste management and dosimetry. Requests for such data are collected on a worldwide basis by the International Atomic Energy Agency into a list called WRENDA (World Request List for Neutron Data Measurements). Another important need is

\section{Contents}

\begin{tabular}{|c|c|c|}
\hline $\begin{array}{l}978 \text { Hewlett-Packard } \\
\text { Europhysics Prize }\end{array}$ & & \\
\hline $\begin{array}{l}\text { Central Bureau for } \\
\text { Nuclear Measuremer }\end{array}$ & nts. & 1 \\
\hline $\begin{array}{l}\text { Neutron Stars in } \\
\text { Binary Systems }\end{array}$ & . & 5 \\
\hline Lattice Dynamics . . & 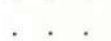 & 9 \\
\hline New President of EPS & . & 11 \\
\hline Computational Physics & & 12 \\
\hline Other Society News. & . & 12 \\
\hline
\end{tabular}


Table 1: Characteristics of the Van de Graaff Accelerator

Ion Energy
(MeV)
1
2
3
5
7

D.C. Current
$(\mu \mathrm{A})$
20
25
30
35
20

Pulse Current (1)
\[ \begin{array}{c}(\mu \mathrm{A}) \\ 3 \\ 4 \\ 6 \\ 6 \\ 6\end{array} \]

Puise Width
(ns)
2.5
1.8
1.5
1.3
1.25

(1) average, at $25 \mathrm{MHz}$ pulse repetition rate. for standards and for the development of methods for precise neutron flux measurements. Results obtained at CBNM are routinely transmitted to the CCDN (Centre de Compilation des Données Neutroniques) at Paris, where data files are being kept and continuously updated.

\section{Facilities}

CBNM has two major installations on which it carries out its neutron data measurement programme: a 7 MV Van de Graaff accelerator and a $150 \mathrm{MeV}$ electron linear accelerator.

The Van de Graaff accelerator is a vertical single stage machine equipped with an ion source for the production of proton, deuteron and helium ion beams and containing a nanosecond klystron bunching system. Its characteristics are shown in Table 1.

Neutrons are produced by the reacor $\mathrm{T}(\mathrm{d}, \mathrm{n})^{4} \mathrm{He}$ with energies ranging from $30 \mathrm{keV}$ to $23 \mathrm{MeV}$.

The linear accelerator is an S-band machine with a coaxial triode gun specially designed for emitting high current electron bursts of short duration, a standing wave buncher and two travelling wave sections. The characteristics are summarized in Table 2 .

To produce intense neutron bursts of short duration which is the aim of the facility, the high current bursts of electrons impinge on a mercury cooled $\mathrm{U}$ target, where the electrons generate bremsstrahlung, which in turn produces fast neutrons by means of $(\gamma, \mathrm{n})$ and $(\gamma, \mathrm{f})$ processes. Two moderators (polyethylene slabs or water in beryllium cans) are used to tions ${ }^{7} \mathrm{Li}(p, n)^{7} \mathrm{Be}, \mathrm{T}(\mathrm{p}, \mathrm{n})^{3} \mathrm{He}, \mathrm{D}(\mathrm{d}, \mathrm{n})^{3} \mathrm{He}$ produce a high neutron flux with roughly a $1 / E$ energy spectrum. Differential neutron cross sections and other data are then measured by applying neutron time-of-flight techniques to determine the neutron energy. For this purpose, 12 evacuated neutron flight paths, with lengths between 8 and $400 \mathrm{~m}$ are installed and equipped with collimators and detector stations.

\section{Neutron Data for Applied Purposes}

The main emphasis in past neutron data measurement programmes has been on differential neutron crosssections and other neutron data which were requested for the development of fission reactors. Thus, the fission cross-sections of the main fissile isotopes and the neutron capture crosssections of fertile isotopes as well as fission products have been measured with high neutron energy resolution. As example we can cite an extensive series of measurements on ${ }^{238} \mathrm{U}$ which have recently been completed with the result that the cross-sections of this most important fertile nucleus are now known to an accuracy of at least $5 \%$. Recently, the Van de Graaff accelerator has been used accurately to measure the spectrum of prompt neutrons after neutron induced fission of ${ }^{235} \mathrm{U}$ and ${ }^{239} \mathrm{Pu}$. In addition, cross-sections for several neutron induced threshold reactions, used for in-pile neutron dosimetry, have been measured.

An essential requirement for the safety of a fast reactor is that its Doppler coefficient be negative, i.e. that the reactivity of the reactor decreases with increasing temperature.
Table 2 : Characteristics of the Linear Accelerator

\begin{tabular}{lccccc} 
& & $\begin{array}{c}\text { Short pulses } \\
(1)\end{array}$ & \multicolumn{3}{c}{ Long pulses } \\
& & $(2)$ & \\
Burst width & $4 \mathrm{~ns}$ & $10 \mathrm{~ns}$ & $100 \mathrm{~ns}$ & $1 \mu \mathrm{s}$ & $2 \mu \mathrm{s}$ \\
Instantaneous currents & $9 \mathrm{~A}$ & $9 \mathrm{~A}$ & $1.5 \mathrm{~A}$ & $0.22 \mathrm{~A}$ & $0.22 \mathrm{~A}$ \\
Repetition frequencies & $900 \mathrm{~Hz}$ & $900 \mathrm{~Hz}$ & $880 \mathrm{~Hz}$ & $380 \mathrm{~Hz}$ & $250 \mathrm{~Hz}$ \\
Electron energies & $120 \mathrm{MeV}$ & $105 \mathrm{MeV}$ & $87 \mathrm{MeV}$ & $100 \mathrm{MeV}$ & $100 \mathrm{MeV}$ \\
Beam power & $3.9 \mathrm{~kW}$ & $8.5 \mathrm{~kW}$ & $11.5 \mathrm{~kW}$ & $11 \mathrm{~kW}$ & $11 \mathrm{~kW}$
\end{tabular}

(1) For short pulses the maximum energy at small currents is $130 \mathrm{MeV}$.

(2) For long pulses the maximum energy at small current is $150 \mathrm{MeV}$.
As the Doppler coefficient depends on the properties of the narrow compound nuclear resonances which are characteristic of the cross-sections of heavy nuclei, the high resolution cross-section data obtained with the linear accelerator are analysed to determine the neutron resonance parameters. Again, the resonance parameters of ${ }^{238} \mathrm{U}$ are particularly important in this respect.

Studies of the higher actinide isotopes which are important for questions related to the build up of transplutonium isotopes in reactors, to their burn-up in reactors and to waste management, will play an important role in the present and future measurement programmes. Also data requested for the design of fusion reactor blankets will be included.

Special emphasis has always been put on the accurate measurement of neutron cross-sections which are regarded as standards. Thus the crosssections for the reactions ${ }^{1} \mathrm{H}(n, n)^{1} \mathrm{H}$, ${ }^{6} \mathrm{Li}(\mathrm{n}, \alpha)^{3} \mathrm{H}, \quad{ }^{10} \mathrm{~B}(\mathrm{n}, \alpha)^{7} \mathrm{Li}, \quad{ }^{197} \mathrm{Au}(\mathrm{n}, \gamma){ }^{198} \mathrm{Au}$ and ${ }^{235} \mathrm{U}(\mathrm{n}, \mathrm{f})$ have been measured, mainly at the Van de Graaff laboratory with an accuracy of typically $3-5 \%$.

\section{Nuclear Structure Information}

It has often been found in physics that an order-of-magnitude improvement in experimental precision results in the discovery of new phenomena and new challenging questions. In the case of neutron data measurements, it is the high energy resolution required for some of the cross-section measurements and available with the linear accelerator time-of-flight spectrometer, that has led to interesting questions and results, in the field of basic nuclear physics. A rough typical figure for the resolution is $\triangle E / E \approx$ $10^{-3}$, where $E$ is the neutron kinetic energy. For $E=1 \mathrm{keV}$ we have $\Delta E=$ $1 \mathrm{eV}$, so that with respect to the excitation energy of the compound nucleus, $\mathrm{U}(=5 \mathrm{MeV})$, this means $\Delta \mathrm{U} / \mathrm{U}$ $\approx 2.10^{-7}$. Thus, by neutron time-offlight spectroscopy, highly excited nuclei may be studied with a resolution hardly attainable by any other means. The figure of $\Delta E=1 \mathrm{eV}$ also compares favourably with the average spacing of compound nuclear levels of a given spin, which is typically, 10 to $100 \mathrm{eV}$ for heavy nuclei (and much larger for lighter nuclei). Thus by neutron resonance spectroscopy, the properties of individual levels at excitation energies corresponding to the neutron binding energy may be studied.

Subjects of interest in the first instance are the statistical properties of 
nuclear levels at high excitation energies, e.g. their average level spacing, neutron and $\gamma$-ray strength functions, and the distribution of individual resonance parameters around their averages. Further, the dependence of such quantities on gross nuclear properties like mass number or nuclear shell structure is also of great interest.

The counterpart to these statistical properties is, of course, the nuclear structure information inherent in nonstatistical effects, i.e. the deviations from the normal statistical behaviour. Non-statistical effects related to the valence nuclear model of neutron radiative capture have recently stimulated wide interest. Particularly interesting have been the investigations following the discovery, at the CBNM linear accelerator laboratory, of intermediate structure in neutron induced fission cross-sections. As these structures are caused by states corresponding to the secondary minimum in the deformation energy potential (the double bump), the study of their properties has yielded information on the detailed shape of that potential, i.e. on the depth of the second minimum and the penetrabilities of the two barriers.

\section{Non-Neutron Nuclear Data Measure-} ments

The programme of the Non-Neutron Nuclear Data Group is mainly concerned with the decay properties of radio-nuclides. They are needed in many branches of nuclear energy, and in industrial, environmental and medical applications of isotopes and radiation. These applications have been discussed in various international committees and the need for more reliable data strongly stressed. To improve the situation, it is necessary to perform detailed evaluations on what is already available and to concentrate on measurements yielding high accuracy and more reliability. Three main lines of activity should be mentioned :

\section{Measurement and Evaluation of Decay Scheme Data}

The half-lives of the important fissile and fertile materials are still not known to the required accuracy. Measurements have been performed especially on long-lived nuclides, as e.g., ${ }^{233} \mathrm{U}$ and ${ }^{239} \mathrm{Pu}$. In such cases, the $\alpha$ activity of the sample is measured by counting methods and the number of atoms is determined by the mass-spectrometric isotope dilution technique. Another important half-life is that of ${ }^{241} \mathrm{Pu}$. There are still discrepancies existing between the values obtained by direct measurements of the ${ }^{24} \mathrm{Pu}$ decay and those using various mass-spectrometric techniques, and in addition between these techniques and those values obtained by the ${ }^{247} \mathrm{Am}$-ingrowth method using $x_{-}$and $;$-ray counting techniques. Measurements on this isotope are in progress. Additionally, $\mathrm{X}$ - and $\%$-ray intensities of the actinides are studied.

Some work is performed to determine $\gamma$ - and $X$-ray intensities, internal conversion data and half-lives of nuclides such as ${ }^{93} \mathrm{Nb}^{\prime \prime \prime},{ }^{103} \mathrm{Rh}^{\mathrm{m}}$ and ${ }^{115} \mathrm{In}^{\mathrm{m}}$ which are formed by neutron induced threshold reactions. Such data are needed for in-pile fluence and spectrum measurements. Other nuclides, such as ${ }^{139} \mathrm{Ce},{ }^{141} \mathrm{Ce}$ or ${ }^{203} \mathrm{Hg}$, which emit a single $\gamma$ ray, are very suitable for intensity calibration of $\because$-ray detectors, provided the decay properties are accurately known. Some effort is devoted to orbital electron capture by the nucleus. The whole field has been reviewed in the light of current understanding of weak interactions in collaboration with American, British and German physicists. Special attention is paid to the determination of electron capture probabilities and their ratios, and of the ratio of electron capture to $\beta^{+}$. emission. Measurements of higher order effects for various radionuclides such, as the internal bremsstrahlung accompanying electron capture transitions, or the total K-shell internal-ionization probabilities during $\beta^{-}$decay, have been carried out.

An extensive compilation of experimental results of internal conversion coefficients and their ratios published later than November 1965 is actually in progress in collaboration with German research workers. The data are treated separately according to the source of experimental information such as : radioactive decays, reaction processes, EO transitions.

\section{Measurement and Evaluation of Atomic Data}

Detailed knowledge of radiationless transition rates and energies is necessary for the interpretation of a large variety of measurements in nuclear and atomic physics. For example, electron capture or internally converted $\dot{\gamma}$ transitions, can only be determined by measuring $X$-ray intensities, and applying pertinent subshell fluorescence yields to derive primary vacancy distributions. Consequently, $X$-ray fluorescence yields, Auger and Coster-Kronig transition probabilities are studied thoroughly. Particular emphasis at present is given to effects originating in L-shell vacancies where
POST

DOCTORAL

RESEARCH POSITION

at the

SWISS FEDERAL

INSTITUTE

of TECHNOLOGY

ETH, ZURICH

A post-doctoral research ap-

pointment is available at

the Laboratory

for High Energy Physics.

Applicants who have experience in Medium Energy Physics should be interested in joining an experimental program at the Swiss Institute of Nuclear Research (SIN), involving experiments in nuclear muon capture and rare decays. A three years appointment is offered with possible renewal.

Applications should contain a complete résumé, a list of publications and the names of three referees.

They should be sent as early as possible to

Dr. H.K. Walter, Laboratory for High Energy Physics ETHZ, c/O SIN, CH-5234 villigen

(Switzerland). 
the data are inadequate or non-existent.

\section{Nuclear Metrology}

Development and improvement of precision measuring techniques are the basis of an accurate determination of nuclear or atomic data. Therefore, part of the effort is devoted to radionuclide metrology. For example, the laboratory participated successfully in intercomparisons, as organized by the Bureau International des Poids et Mesures (BIPM), Paris, during the last year to determine the radioactive concentration of solutions of ${ }^{57} \mathrm{Co}$ and ${ }^{139} \mathrm{Ce}$ and to assess experimentally the validity of approximations made in coincidence counting, using ${ }^{60} \mathrm{Co}$ sources with activities ranging from $10^{3}$ to $10^{5} \mathrm{~Bq}$.

\section{Materials Research}

The Materials Activity at the CBNM can be broadly divided into three parts, namely; sample preparation, mass spectrometry and other analytical techniques. Historically the facilities were almost wholly used for the fabrication and analysis of well defined samples and targets to be used in nuclear research. However, in recent years, the CBNM has applied the expertise it has gained in the nuclear field to broaden its scope and provide samples for non-nuclear research.

\section{Sample Preparation}

Samples are provided in many diverse forms such as thin layers both supported and self supporting (e.g. of fissile materials), rolled foils (for example, of metals for neutron flux measurements) and discs and rods of certified alloys. Samples in solution and powder form are also available.

The methods of preparation of these samples are wide ranging and include amongst them: vacuum evaporation both by electron beam bombardment and resistive heating, cathodic sputtering, levitation melting which permits the very homogeneous mixing of alloys of different metals, and electrospraying where, for example, very uniform layers of compounds of nearly every element can be provided. Typical samples deposited by the last method are radioactive $\mathrm{Th}, \mathrm{Np}, \mathrm{Am}$ and other transplutonium elements.

In addition, the facilities exist to carry out, in vacuo, the rolling and cutting of disc plate and rod samples so that no additional contaminants are introduced during the forming.

\section{Analytical Techniques}

The analytical laboratories where assay and certification of many of these samples are carried out, has at its disposal all the facilities necessary for the precise determinations required. In the metrology section, numerous precision balances are available for weighings in the sub microgram range. All the weighings are related (directly) to primary standards. Several of the balances are built into ultra high vacuum evaporation units such that the weighings of thin layers, for the highest precision and minimum of contamination, are made in situ and during deposition. Classical quantities such as density, length, temperature and pressure can also be measured with extreme precision.

Thickness and uniformity of thin layers are determined by a variety of techniques including densitometers, interferometers, X-ray fluorescence, $X$-ray radiography, $\beta$ absorption and physical profiling by a stylus (Talystep). Recently, the alpha absorption technique has been used to measure the homogeneity of large area self supporting foils of less than $20 \mu \mathrm{g} / \mathrm{cm}^{2}$. Rutherford back-scattering of alphas and protons provided at the $3 \mathrm{MeV}$ Van de Graaff accelerator have also been introduced as a means of characterising layered structures and alloys non- destructively to depths of several micrometers. Work is in progress to add proton induced $\mathrm{X}$-rays and nuclear-reaction spectrometry to this powerful tool for quantitative analysis of trace impurities in the ppm to $\mathrm{ppb}$ range. Auger spectrometry and scanning electron microscopy are available to complement the other methods. Classical and high precision chemical methods are at hand together with electrochemical techniques such as coulometry, polarography, emission and absorption spectrometry and spectrophotometry to obtain accurate analysis of small quantities of elements or purified isotopes. These activities were primarily concentrated on the accurate analyses of substances of interest to the nuclear industry and research, such as $\mathrm{U}, \mathrm{Pu}, \mathrm{Li}$ and the transplutonic elements, but in recent times, interest has broadened to include analyses of materials for non nuclear applications. For example, studies have been made of an elemental analysis of electrolytic copper, zinc and lead ores and of carbon and nitrogen gas concentrations in metals and compounds. Much of this work is done in collaboration with the Bureau Communautaire de Référence (BCR) of the Communities.

\section{Mass Spectrometry}

The mass spectrometry laboratory performs accurate analysis of the isotopic composition of solids and gases, mainly materials of interest to the nuclear energy field such as $\mathrm{Li}, \mathrm{B}$, and fissile materials such as $U$ and $\mathrm{Pu}$.

On request, the laboratory provides other institutes with analyses of nuclear fuel, burn-up determinations, isotopic assaying of reference samples, quantitative analyses of gases, including fission gases and high precision isotope ratio determinations. To give an idea of the accuracy of this technique, samples have been measured for their ${ }^{235} \mathrm{U} /{ }^{238} \mathrm{U}$ ratio and certified with a precision of $\pm 0.2 \%$, the reproducibility of these measurements being better than $0.1 \%$.

The laboratory is also concerned with instrumental development, compilations in connection with atomic weights and studies on mass discrimination and isotopic dilution analysis developments. In connection with this, since 1969, the CBNM has taken an active part in the development of techniques and in providing scientific and technical support to the Safeguards Directorate of the Commission of the Communities whose task it is to safeguard fissile material in the Community by virtue of the Rome Euratom Treaty. During inspection of the fuel elements, samples are taken from the various nuclear installations within the Community and these must be analysed for fissile material content and composition. In general the CBNM provides isotopic composition and element assay by isotopic dilution mass spectrometry and these results are compared with those declared by the primary fuel users.

\section{Conclusion}

The work of the CBNM is organised into three main areas :

- Neutron metrology for the measurement of neutron cross sections and standards :

- Nuclear metrology associated with the decay of radionuclides and measurement of isotopic composition ;

- Preparation and characterisation of reference materials and surfaces required for nuclear and to some extent non-nuclear measurements. In addition, the CBNM provides a service to the Community by making available its expertise both giving advice and providing positive laboratory support to outside requesters.

Finally, the CBNM provides facilities and technical resources for students and Euratom grant holders from member countries to carry out their research studies within the laboratories, which they may use in partial fulfillment of their thesis requirements. 